\title{
Análisis epidemiológico de pacientes coinfectados con enfermedad de Chagas y cisticercosis
}

\author{
Ana Carolina Faleiros ${ }^{1}$, Ruy Lino-Junior ${ }^{2}$, Virlanea Lima ${ }^{3}$, Camila Cavellani, \\ Rosana Rosa Corrêa ${ }^{3}$, Mauricio Llaguno ${ }^{4}$, Marlene Reis ${ }^{3}$, Vicente Teixeira ${ }^{3}$
}

\footnotetext{
1 Disciplina de Biología Celular, Universidad Federal del Triángulo Mineiro, Uberaba, Minas Gerais, Brasil 2 Instituto de Patología Tropical y Salud Pública, Universidad Federal de Goiás, Goiânia, Goiás, Brasil 3 Disciplina de Patología General, Universidad Federal del Triángulo Mineiro, Uberaba, Minas Gerais, Brasil ${ }^{4}$ Posgraduación de Medicina Tropical, de la Universidad Federal del Triángulo Mineiro, Uberaba, Minas Gerais, Brasil
}

Introducción. Se ha sugerido que la infección por larvas de Taenia solium (cisticercosis) podría inducir un estado de inmunosupresión en el huésped, aumentando el riesgo de adquirir infecciones múltiples después de estar expuesto a otros parásitos, entre ellos Trypanosoma cruzi, particularmente entre habitantes de áreas endémicas en países en desarrollo.

Objetivos. Evaluar un posible aumento en la presentación endémica de infección por T. cruzi (enfermedad de Chagas) en pacientes con cisticercosis.

Materiales y métodos. Se estudiaron 1.501 autopsias de individuos mayores de 15 años de edad del Hospital Universitario de Uberaba, Minas Gerais, Brasil, 1970-2004. Se dividieron los casos en cuatro grupos: (1) sin infección, (2) con cisticercosis, (3) con enfermedad de Chagas y (4) coinfectados con cisticercosis y enfermedad de Chagas. Se analizaron las variables de raza, sexo y edad.

Resultados. Más de la mitad de todos los casos no presentaban infección (848 casos o 56,5\%); 611 pacientes $(40,7 \%)$ tenían enfermedad de Chagas; 72 casos $(4,8 \%$ de las autopsias) tenían cisticercosis y $30(41,7 \%)$ presentaron coinfección de cisticercosis con enfermedad de Chagas. La raza blanca y el sexo masculino fueron predominantes en todos los grupos. La mediana de edad más joven fue encontrada en el grupo sin infección (46 años), seguido por aquéllos que presentaban enfermedad de Chagas (49 años) y por aquéllos con coinfección de cisticercosis y enfermedad de Chagas (57,5 años).

Conclusión. En teoría, todos los pacientes tuvieron un riesgo similar de exposición para ambos parásitos. Sin embargo, este trabajo demuestra que, en la población estudiada, la enfermedad de Chagas fue, aproximadamente, 10 veces más frecuente en los casos coinfectados con cisticercosis.

Palabras clave: enfermedad de Chagas/epidemiología, cisticercosis, infección, supervivencia, autopsia.

\section{Epidemiological analysis of patients coinfected with Chagas disease and cysticercosis}

Introduction. Among inhabitants of endemic areas in the developing world, infection with the larva of Taenia solium (cysticercosis) may possibly induce a state of immunosuppression in the host, thereby increasing the risk of multiple infections after exposure to other parasites, such as Trypanosoma cruzi.

Objective. Increase in the epidemiological occurrence of infection was assessed for Trypanosoma cruzi (Chagas disease) in patients with cysticercosis.

Materials and methods. At the University Hospital in Uberaba, Minas Gerais, Brazil, data were obtained from autopsies performed between 1970-2004 on 1,501 subjects older than 15 years of age. Cases were divided into four groups: (1) no infection, (2) cysticercosis only, (3) Chagas disease only, and (4) cysticercosis coinfected with Chagas disease. Race, gender and age data were analyzed.

Results. More than half of the cases showed no infection (848 cases or $56.5 \%$ ); Chagas disease was found in 611 patients $(40.7 \%)$; 72 cases (4.8\% of the autopsies) had cysticercosis and 30 of them $(41.7 \%)$ were co-infected with Chagas disease. White race and male gender 
were predominant in all groups. The youngest median age was found in the non-infected group (46 years), followed by those with Chagas disease without cysticercosis (49 years) and those coinfected with cysticercosis and Chagas disease ( 57.5 years).

Conclusion. Presumably, all patients had a similar exposure to both parasites. However, in this study population, Chagas disease was approximately 10 times more frequent in patients coinfected with cysticercosis.

Key words: Chagas disease/epidemiology, cysticercosis, infection, survivorship (Public health), autopsy.

Gran parte de la población mundial, en especial en los países subdesarrollados, está expuesta a condiciones sanitarias inadecuadas, lo que la hace más susceptible a infecciones por dos o más patógenos (1).

La infección inicial por un parásito extracelular puede llevar a alteraciones de la susceptibilidad a parásitos intracelulares. Sin embargo, esas modificaciones dependen de la evolución de la infección primaria (2). Eso ocurre, por ejemplo, en el caso de coinfección entre Schistosoma mansoni y Salmonella typhi (3), S. mansoni y Plasmodium falciparum (4), Ascaris lumbricoides y P. falciparum (5), Babesia microti y Fasciola hepatica (6), Trypanosoma cruzi y cisticerco de la Taenia crassiceps (2).

Estudios previos en poblaciones humanas mostraron la asociación de la neurocisticercosis con gliomas (7), enfermedades hematológicas (8) y encefalitis japonesa (9), y como factor de riesgo para los procesos isquémicos (10). Además, existen algunos informes de casos de pacientes coinfectados con cisticercosis y $\mathrm{VIH}$ y en un estudio esta coinfección se asoció como principal causa básica de muerte (11). Sin embargo, poco se sabe sobre la influencia de la infección por VIH en la frecuencia y el curso clínico de la neurocisticercosis (12).

En la cisticercosis, las manifestaciones son inespecíficas y varían según el sitio anatómico de la infección, el número y las características de

\footnotetext{
Correspondencia:

Ana Carolina Guimarães, Departamento de Ciências Biológicas, Disciplina de Biologia Celular, Universidade Federal do Triângulo Mineiro, Rua Frei Paulino \#30, CEP: 38025-180, Uberaba, Minas Gerais, Brasil carolinafaleiros@dcb.uftm.edu.br

Recibido: 02/04/08; aceptado:31/10/08
}

las lesiones, las fases de desarrollo del parásito y la intensidad de la respuesta inmune inflamatoria del huésped frente al cisticerco (13).

En la enfermedad de Chagas, múltiples factores actúan directa e indirectamente en la aparición de las lesiones que surgen en los diferentes órganos. Algunos son inherentes a los parásitos y otros se relacionan con el huésped. Entre los factores determinantes de la enfermedad de Chagas, debe considerarse el inóculo inicial, las reinfecciones, las características biológicas de las cepas, los clones de T. cruzi y la respuesta inmune del huésped a nivel celular y humoral $(14,15)$.

Los estudios experimentales han demostrado que la infección primaria por $T$. crassiceps puede modificar la susceptibilidad a $T$. cruzi. Este hecho podría estar relacionado con una fuerte respuesta de tipo Th2, la cual produciría alteraciones inmunes induciendo algún tipo de protección asociada a una producción inespecífica de interferón gamma $(2,16)$.

Los pacientes sometidos a autopsia en el Hospital Escuela de la Universidad Federal del Triangulo Mineiro, Uberaba, Minas Gerais, Brasil, provenían de un área endémica tanto para enfermedad de Chagas como para cisticercosis, y podían tener una mayor probabilidad de presentar esta coinfección.

El objetivo de este trabajo fue evaluar un posible aumento en la presentación epidemiológica de infección por $T$. cruzi en pacientes con cisticercosis.

\section{Materiales y métodos \\ Criterios de selección}

Se revisaron 2.735 protocolos de autopsias realizadas en el Hospital Escuela de la 
Universidad Federal del Triángulo Minero, Uberaba, Minas Gerais, en el período de 1970 a 2004. Se excluyeron los protocolos incompletos y los de pacientes con edad inferior a 15 años (límite inferior del grupo con cisticercosis). Se obtuvieron 1.501 protocolos, divididos en cuatro grupos: coinfectados con cisticercosis y enfermedad de Chagas; con cisticercosis sin enfermedad de Chagas; con enfermedad de Chagas sin cisticercosis, y, sin cisticercosis ni enfermedad de Chagas.

\section{Datos recolectados}

Se registró información relativa a la presencia, el sitio anatómico y la etapa evolutiva del cisticerco: vesicular, vesicular coloidal, granular nodular y nodular calcificado.

En relación con la enfermedad de Chagas se registraron: serología positiva para $T$. cruzi, manifestaciones clínicas, presentación de otros tipos de cardiopatías diferentes de la chagásica (hipertensiva, reumática e isquémica) y las variables edad, raza (blanco o no) y sexo.

\section{Criterios de diagnóstico}

Los criterios para el diagnóstico de cisticercosis humana se siguieron de acuerdo con métodos previamente determinados por otros autores (17). Se utilizó el criterio absoluto que tiene en cuenta la demostración histológica o la visualización directa del parásito obtenido del material de autopsia.

Para el diagnóstico de enfermedad de Chagas, se tomó en consideración la existencia de dos serologías positivas o de manifestaciones específicas como cardiopatía chagásica o visceromegalias.

\section{Análisis estadístico}

Para el análisis estadístico se elaboró una planilla electrónica. Se probaron las variables para verificar si presentaban distribución normal, por medio de la prueba de Kolmogorov-Smirnov, y también, la homogeneidad de la muestra. Como la distribución no fue normal, se utilizaron pruebas no paramétricas en la comparación entre dos grupos, la prueba de Mann-Whitney, y en la comparación entre tres o más grupos, la prueba de Kruskal-Wallis, cuando fue necesario, seguida de la prueba de Dunn. Las proporciones se compararon por la prueba de ji al cuadrado, acompañada de la prueba exacta de Fisher. $\mathrm{Se}$ consideraron como estadísticamente significativos los valores de $p$ menores de $5 \%$ $(\mathrm{p}<0,05)$.

\section{Aspectos éticos}

Este estudio fue aprobado por el Comité de Ética en Investigación de la Universidad Federal del Triángulo Mineiro.

\section{Resultados}

De los 1.501 protocolos de autopsias revisados, se encontraron 848 casos $(56,5 \%)$ de pacientes no infectados y 581 casos $(40,7 \%)$ con enfermedad de Chagas sin cisticercosis. Hubo 72 casos $(4,8 \%)$ de cisticercosis, de los cuales, 30 $(41,7 \%)$ estaban coinfectados con enfermedad de Chagas. Cuarenta y dos casos $(58,3 \%)$ presentaron cisticercosis sin enfermedad de Chagas $\left(\chi^{2}=0,0104 ; p=0,919\right)$.

De los 30 casos del grupo coinfectado con cisticercosis y enfermedad de Chagas, 26 $(86,7 \%)$ presentaron la forma cardiaca de la enfermedad de Chagas, siete $(23,3 \%)$ la forma digestiva (visceromegalias) y dos $(6,7 \%)$ la forma indeterminada. De los 72 casos de cisticercosis, $17(23,6 \%)$ tuvieron cisticercosis cardiaca y 8 casos $(26,7 \%)$ presentaron concomitantemente enfermedad de Chagas ( $\left.\chi^{2}=0,0550 ; p=0,815\right)$. Los otros casos de cisticercosis presentaron localización encefálica o músculo-esquelética.

La raza blanca y el sexo masculino predominaron en todos los grupos (cuadro 1). Las medianas de la edad en los grupos de coinfectados con cisticercosis y enfermedad de Chagas ( 57,5 años) y en el grupo con enfermedad de Chagas sin cisticercosis (49 años), fueron significativamente superiores a la del grupo sin cisticercosis ni enfermedad de Chagas (46 años) $(H=14,477$; $p<0,05)$.

La etapa vesicular y la nodular calcificada se presentaron solamente en el grupo con cisticercosis sin enfermedad de Chagas (25\% para ambos). La etapa vesicular se observó tanto 
en el grupo de coinfectados con cisticercosis y enfermedad de Chagas, como en el grupo con cisticercosis sin enfermedad de Chagas $(80 \%$ y $37,5 \%$, respectivamente). La etapa granular nodular se observó en mayor número en el grupo coinfectado con cisticercosis y enfermedad de Chagas (20\%), comparado con el grupo con cisticercosis sin enfermedad de Chagas (12,5\%) $\left(\chi^{2}=3,645 ; p=0,302\right)$. Cuando se agruparon las etapas en estadios iniciales (etapas vesicular y vesicular coloidal) y estadios finales (etapa granular nodular y nodular calcificada), se observó que, tanto en el grupo coinfectado con cisticercosis y enfermedad de Chagas como en el de cisticercosis sin enfermedad de Chagas, prevalecieron los estadios iniciales $(83,3 \%$ y $57,2 \%$, respectivamente) $(p=0,559)$.

Con respecto a la presencia de otros tipos de cardiopatía en los 35 casos analizados, se encontró que, en el grupo coinfectado con cisticercosis y enfermedad de Chagas, 17\% presentó cardiopatía hipertensiva, 33\% cardiopatía reumática y $17 \%$ cardiopatía isquémica. En el grupo con cisticercosis sin enfermedad de Chagas, 22\% presentó cardiopatía hipertensiva y $33 \%$ cardiopatía isquémica. En el grupo con enfermedad de Chagas sin cisticercosis, 20\% presentócardiopatíahipertensiva,20\%cardiopatía reumática y $30 \%$ cardiopatía isquémica. En el grupo sin cisticercosis ni enfermedad de Chagas, $40 \%$ presentó cardiopatía hipertensiva, $10 \%$ cardiopatía reumática y $40 \%$ cardiopatía isquémica.

\section{Discusión}

La región del Triángulo Mineiro es altamente endémica tanto para enfermedad de Chagas como para cisticercosis, en comparación con otras regiones brasileras $(18,19)$. No encontramos una mayor frecuencia de enfermedad de Chagas entre los pacientes con cisticercosis $(41,7 \%)$. Sin embargo, ese porcentaje fue superior al de enfermedad de Chagas en el grupo sin cisticercosis $(40,7 \%)$. Estos resultados demuestran que la concomitancia de dos o más parasitosis no es un hecho inusual, principalmente cuando se trata de habitantes de áreas endémicas.

En este estudio verificamos una frecuencia de cisticercosis de $4,7 \%$, mayor a la observada en otros estudios realizados anteriormente en material semejante, en los cuales la frecuencia de cisticercosis varió de 2,4\% a 3,3\% $(18,20)$.

Se encontró un predominio del sexo masculino y de la raza blanca, lo que concuerda con otros autores $(18,21)$. A pesar de que se ha reportado que la enfermedad de Chagas es más frecuente en mujeres, su letalidad es mayor en el sexo masculino y en personas de razas diferentes a la blanca (22).

Los pacientes que presentaron coinfección con cisticercosis y enfermedad de Chagas tuvieron mayor tiempo de supervivencia que los que presentaron infección sólo por cisticercosis o por enfermedad de Chagas y que aquéllos sin infección.

Cuadro 1. Distribución por sexo y raza de 1.501 pacientes sometidos a autopsia, entre los grupos coinfectados con cisticercosis y enfermedad de Chagas, con cisticercosis sin enfermedad de Chagas, con enfermedad de Chagas sin cisticercosis, sin cisticercosis ni enfermedad de Chagas.

\begin{tabular}{lcccc}
\hline Sexo* $^{*}$ & $\begin{array}{c}\text { Con CC y EC } \\
\mathbf{n}(\%)\end{array}$ & $\begin{array}{c}\text { Con CC sin EC } \\
\mathbf{n}(\%)\end{array}$ & $\begin{array}{c}\text { Con EC sin CC } \\
\mathbf{n}(\%)\end{array}$ & $\begin{array}{c}\text { Sin CC ni ECn } \\
\mathbf{n}(\%)\end{array}$ \\
\hline Masculino & $22(73,4 \%)$ & $24(57,2 \%)$ & $397(68,4 \%)$ & $537(63,4 \%)$ \\
Femenino & $8(26,6 \%)$ & $18(42,8 \%)$ & $184(31,6 \%)$ & $311(36,6 \%)$ \\
Raza** & & & & \\
Blanco & $16(53,3 \%)$ & $29(69 \%)$ & $349(60 \%)$ & $522(61,5 \%)$ \\
No blanco & $14(46,7 \%)$ & $13(31 \%)$ & $232(40 \%)$ & $326(38,5 \%)$ \\
Total & $\mathbf{3 0 ( 1 0 0 \% )}$ & $\mathbf{4 2 ( 1 0 0 \% )}$ & $\mathbf{5 8 1 ( 1 0 0 \% )}$ & $\mathbf{8 4 8 ( 1 0 0 \% )}$ \\
\hline
\end{tabular}

*Prueba de ji al cuadrado=5,901; $p=0,117$

${ }^{* *}$ Prueba de ji al cuadrado $=2,207 ; p=0,531$

EC: enfermedad de Chagas; CC: cisticersosis 
Los cisticercos y T. cruzi pueden desencadenar modulación del sistema inmuneque, pormecanismos distintos, llevarían a alteraciones en la progresión de la enfermedad en el huésped $(23,24)$.

Las citocinas son los principales factores involucrados en la comunicación entre las células $\mathrm{T}$, los macrófagos y otras células inmunes (25). El factor del metacéstodo induce un descenso en la regulación Th1, Th2 y de citocinas proinflamatorias de modo inespecífico. La supresión en la producción de FNT- $\alpha$, provocada por la estimulación de macrófagos indicaría un mecanismo de inhibición inflamatoria, ocasionado por el factor del metacéstodo por medio de una regulación por disminución de la producción de citocinas proinflamatorias (23).

T. cruzi puede promover alteraciones tanto de la respuesta inmune humoral como de la celular. La exposición de los linfocitos activados por $T$. cruzi llevaría a una disminución de la producción de IL-2 y expresión de sus receptores, los cuales estarían directamente relacionados con la progresión del ciclo celular de linfocitos activados (26-28).

La inducción del tipo de respuesta inmune depende de la localización del parásito, intracelular o extracelular, lo cual estimularía diferentes tipos de respuesta celular que pueden llevar a control o a progresión de la enfermedad $(23,24)$.

Existen varios informes en la literatura que indican la coexistencia de dos o más patógenos en un mismo individuo. En algunos de esos casos ocurre un cuadro peculiar en que la progresión de la enfermedad sigue un desarrollo favorable al huésped (3-6). Así, la agresión al tejido del huésped como, por ejemplo, la fibrosis y la inflamación, podría ocurrir de forma menos grave, permitiendo una mayor supervivencia, como se observó en nuestro material.

Por otro lado, el grupo sin cisticercosis ni enfermedad de Chagas presentó otros tipos de cardiopatías, diferentes de la chagásica, las cuales estarían actuando de forma decisiva en el desarrollo de lesiones cardíacas graves y, consecuentemente, determinarían un menor tiempo de supervivencia.
En teoría, todos los pacientes tuvieron riesgo de exposición similar para ambos parásitos. Sin embargo, este trabajo muestra que en la población estudiada la enfermedad de Chagas fue, aproximadamente, 10 veces más frecuente que la cisticercosis.

\section{Agradecimientos}

Los autores agradecen a los funcionarios de la Disciplina de Patología General de la Universidad Federal del Triângulo Mineiro por el apoyo técnico en la realización de ese trabajo.

\section{Conflicto de intereses}

Los autores manifiestan no tener conflicto de interés con respecto a los resultados de esta investigación.

\section{Financiación}

Coordenação de Aperfeiçoamento de Pessoal de Nível Superior (CAPES), Conselho Nacional de Desenvolvimento Científico e Tecnológico (CNPq), Fundação de Amparo à Pesquisa do Estado de Minas Gerais (FAPEMIG).

\section{Referencias}

1. Maizels RM, Bundy DA, Selkirk ME, Smith DF, Anderson RM. Immunological modulation and evasion by helminth parasites in human population. Nature. 1993;365:797-805

2. Rodriguez M, Terrazas LI, Márquez R, Bojalil R. Susceptibility to Trypanosoma cruzi is modified by a previous non-related infection. Parasite Immunol. 1999;21:177-85.

3. Muniz-Junqueira MI, Tavares-Neto J, Prata A, Tosta CE. Antibody response to Salmonella typhi in human schistosomiasis mansoni. Rev Soc Bras Med Trop. 1996;29:441-5.

4. Booth M, Vennervald BJ, Butterworth AE, Kariuki HC, Amaganga C, Kimani G, et al. Exposure to malaria affects the regression of hepatosplenomegaly after treatment for Schistosoma mansoni infection in Kenyan children. BMC Med. 2004;2:36.

5. Nacher M, Gay F, Singhasivanon P, Krudsood S, Treeprasertsuk S, Mazier D, et al. Ascaris lumbricoides infection is associated with protection from cerebral malaria. Parasite Immunol. 2000;22:107-13.

6. Fagbeni BO, Christensen NO, Nansen P. Supression of Babesia microti infection in mice concurrently infected with Fasciola hepatica. Vet Parasitol. 1985;17:101-10. 
7. Del Brutto $\mathrm{OH}$, Castillo PR, Mena IX, Freire AX. Neurocysticercosis among patients with cerebral gliomas. Arch Neurol. 1997;54:1125-8.

8. Herrera LA, Benita-Bordes A, Sotelo J, Chávez L, Olvera J, Rascón A, et al. Possible relationship between neurocysticercosis and hematological malignancies. Arch Med Res. 1999;30:154-8.

9. Handique SK, Das RR, Saharia B, Das P, Buragohain R, Saikia P. Coinfection of Japanese encephalitis with neurocysticercosis: an imaging study. Am J Neuroradiol. 2008;29:170-5.

10. Carod-Artal FJ. Strokes caused by infection in the tropics. Rev Neurol. 2007;44:755-63.

11. Santo AH. Cysticercosis-related mortality in the State of São Paulo, Brazil, 1985-2004: a study using multiple causes of death. Cad Saude Publica. 2007;23:2917-27.

12. Delobel P, Signate A, El Guedj M, Couppie P, Gueye $M$, Smadja D, et al. Unusual form of neurocysticercosis associated with HIV infection. Eur $J$ Neurol. 2004;11:55-8.

13. Sotelo J, Del Brutto OH. Brain cysticercosis. Arch Med Res. 2000;31:3-14.

14. Prata A. Chagas' disease. Infect Dis Clin North Am. 1994;8:61-76.

15. Yoshida N. Molecular basis of mammalian cell invasion by Trypanosoma cruzi. An Acad Bras Ciênc. 2006;78:87111

16. Cardillo F, Voltarelli JC, Reed SG, Silva JS. Regulation of Trypanosoma cruzi infection in mice by gamma interferon and interleukin 10: role of NK cells. Infect Immun. 1996;64:128-34.

17. Del Brutto OH, Wadia NH, Dumas M, Cruz M, Tsang VC, Schantz PM. Proposal of diagnostic criteria for human cysticercosis and neurocysticercosis. J Neurol Sci. 1996;142:1-6.

18. Lino RS Jr, Reis MA, Teixeira VP. Occurrence of encephalic and cardiac cysticercosis (Cysticercus cellulosae) in necropsy. Rev Saude Publica. 1999; 33: 495-8.

19. Ramirez LE, Machado MI, Maywald PG, Matos A, Chiari E, Silva EL. Primeira evidência de Trypanosoma rangeli no sudeste do Brasil, região endêmica para doença de Chagas. Rev Soc Bras Med Trop. 1998;31:99-102.

20. Gobbi H, Adad SJ, Neves RR, Almeida HO. Ocorrência de cisticercose (Cysticercus Cellulosae) em pacientes necropsiados em Uberaba, MG. Rev Patol Trop. 1980;9:51-9.

21. Del Brutto $\mathrm{OH}$, Sotelo J. Neurocysticercosis: an update. Rev Infect Dis. 1988;10:1075-87.

22. Dias E, Laranja FS, Miranda A, Nobrega G. Chagas' disease; a clinical epidemiologic and pathologic study. Circulation. 1956;14:1035-60.

23. Arechavaleta F, Molinari JL, Tato P. A Taenia solium metacestode factor nonspecifically inhibits cytokine production. Parasitol Res. 1998;84:117-22.

24. Sher A, Coffman RL. Regulation of immunity to parasites by $\mathrm{T}$ cells and $\mathrm{T}$ cell-derived cytokines. Annu Rev Immunol. 1992;10:385-409.

25. Belardelli F. Role of interferons and other cytokines in the regulation of the immune response. APMIS. 1995;10:161 79

26. Kierszenbaum F, Sztein MB. Mechanisms underlying immunosuppression induced by Trypanosoma cruzi. Parasitol Today. 1990;6:261-4.

27. Kierszenbaum F, Moretti E, Sztein MB. Molecular basis of Trypanosoma cruzi-induced immunosuppression. Altered expression by activated human lymphocytes of molecules which regulate antigen recognition and progression through the cell cycle. Biol Res. 1993;26:197207.

28. Sztein MB, Kierszenbaum F. Mechanisms of development of immunosuppression during Trypanosoma infections. Parasitol Today. 1993;9:424-8. 